\title{
Inhibition of TLR4/TRIF signaling with Dynasore improves Treg/Th17 cell ratio in mice with induced bronchial asthma
}

\author{
SONG-QUAN WU ${ }^{l}$, GUANG-LI WANG ${ }^{l}$,WEI LIANG ${ }^{2 *}$, DAN-LI WANG ${ }^{l}, R U-H U I$ YANG ${ }^{l}$ \\ ${ }^{1}$ Medical College of Lishui University, Lishui, PR China \\ ${ }^{2}$ The People's Hospital of Lishui City, PR China
}

\begin{abstract}
Toll-like receptor 4 (TLR4) and increases in Th17 cells have been associated with inflammatory response in bronchial asthma. To determine if altered $T$ cell profiles are present in asthma, and whether inhibition of TLR4 signaling can ameliorate inflammation in this disease, we treated mouse models of asthma with the TLR4/TRIF signaling inhibitor Dynasore. Asthma was induced in twenty mice by challenge with ovalbumin (OVA). Mice were equally and randomly divided into an asthma model group (AST) or a Dynasore treatment group (DYN). Ten additional mice were treated with saline to serve as a control group $(C T)$. Bronchoalveolar lavage fluid $(B A L F)$ was collected to determine total white and differential cell counts as well as for ELISA to measure secretion levels of cytokines transforming growth factor $\beta 1$ (TGF- $\beta 1$ ), interleukin (IL) 10 (anti-inflammatory) and IL-17 (pro-inflammatory). Asthma model group mice had higher total and differential cell counts, higher amounts of TGF- $\beta 1$ and IL-17, lower amounts of IL-10, and a higher expression of TLR4 than CT mice, which indicated inflammation. The ratio of Treg/Th17 cells was also significantly decreased in AST mice $(p<0.05)$. However, mice with asthma that were treated with Dynasore exhibited improvements in all of these measures compared with AST mice: decreased cell counts, increased IL-10, decreased IL-17 and TGF- $\beta 1$, decreased TLR4 expression, and an increased ratio of Treg/Th17 cells were observed $(p<0.05)$, indicating that inhibition of TLR4 signaling improved inflammatory markers. Thus, TLR4/TRIF signaling may play an important role in pathogenesis of asthma by promoting an imbalance of Treg/Th17 cells.
\end{abstract}

Key words: TLR4/TRIF, asthma, Treg cell, Th17, ovalbumin, Dynasore.

(Centr Eur J Immunol 2013; 38 (4): 454-460)

\section{Introduction}

Bronchial asthma is characterized by chronic inflammation of the airway. The combined action of various inflammatory cells causes hyper-responsiveness and airflow limitation [1]. One type of inflammatory cells, the T lymphocytes, is critical to the occurrence of this condition. In particular, an imbalance in two sub-types of $\mathrm{T}$ lymphocytes, regulatory $\mathrm{T}$ (Treg) and helper $\mathrm{T}$ (Th17) cells, can initiate pathogenesis of asthma, as well as other immune diseases [2,3]. Treg cells are anti-inflammatory cells responsible for immune tolerance and suppression of immune responses. In contrast, Th17 cells are pro-inflammatory and can promote immune responses. Understanding how these imbalances arise could provide much-needed insights into the pathogenesis of asthma.

A critical signaling pathway in immune response involves toll-like receptor 4 (TLR4). This protein, a bridge between innate and acquired immunity, functions in signal transduction during acute inflammatory reactions. Toll-like receptor 4 is also closely related to inflammation and remodeling of the airway in diseases like asthma [4]. Activated TLR4 is transported into cells via two pathways, one dependent on myeloid differentiation factor 88 (MyD88) and one independent of MyD88 [5, 6]. In the MyD88-independent pathway, TLR4 acts as the major junction for signal transduction. An adapter protein, TIR-domain-containing adapter-inducing interferon- $\beta$ (TRIF), coordinates the TLR4 downstream cascade [7]. In asthma, the TLR4/ TRIF pathway participates in the activation, differentiation, and cytokine production of T cells $[8,9]$. However, studies of inflammatory response have demonstrated that blocking the TRL4 signaling cascade by inhibiting TRIF activation diminishes the downstream inflammatory reaction $[10,11]$. Thus, TRIF and TLR4/TRIF signaling are potential targets for asthma therapy. 
Here, inhibition of TLR4/TRIF signaling was investigated in a mouse model of asthma. Specifically, the TLR4/ TRIF-inhibiting agent - Dynasore was introduced in these mice to investigate whether TLR4/TRIF signal inhibition alters the balance of Treg/Th17 cells. Further, we assessed changes in leukocyte numbers and secretion of interleukins (IL-10 and IL-17) in the bronchoalveolar lavage fluid (BALF). The findings could open new avenues for understanding asthma airway inflammation and for developing better asthma therapies.

\section{Material and methods}

\section{Laboratory apparatus}

Polymerase Chain Reaction (PTC-100, MJResearch, USA); Gel Imaging System (UVP, USA); Electric Atomizer (GSK, UK); Microplate Reader (AI, USA); Optical Microscope (Olympus, Japan); Flow Cytometry (FACS Calibur type, Becton Dickinson, USA).

\section{Mouse model of asthma}

Thirty pathogen-free, female BALB/c mice, 6-8 weeks old and 18-20 g body weight, were obtained from the Shanghai SLAC Laboratory Animal Center (Chinese Academy of Sciences).

The mice were housed under specific pathogen-free conditions at $24 \pm 2^{\circ} \mathrm{C}$ and $55 \pm 5 \%$ relative humidity and maintained on a 12-hour light-dark cycle. A commercial diet and potable water were available ad libitum. All experiments described in this study were approved by the Animal Research Ethics Board of the Lishui University (Lishui, Zhejiang Province, China). Mice were randomly divided into 3 groups with 10 mice each: the asthma group (AST group), Dynasore treatment group (DYN group), and the control group (CT group). Asthma was modeled as follows: on days 0 and 12 mice were administered ovalbumin (OVA) (V class, Sigma, USA) in $0.2 \mathrm{ml}$ solution of aluminum hydroxide gel (containing $60 \mu \mathrm{g}$ of OVA and $2.25 \mathrm{mg}$ of Aluminum Hydroxide $\mathrm{Gel}$ ) by intraperitoneal (i.p.) injection. Beginning on day 19, mice received an ultrasonic nebulization of 5\% OVA mixed with $8 \mathrm{ml}$ of the solution, 30 min per day for 5 days. Mice in the DYN group received i.p. injection of $0.2 \mathrm{ml}$ of Dynasore $(1 \mathrm{mg} /$ kg, D7693, Sigma, USA) before OVA nebulization. The CT group received saline instead of OVA treatments.

\section{Preparation of tissue samples and bronchoalveolar lavage fluid}

Fix the mice after their last motivation for $24 \mathrm{~h}$, blood samples were collected retro-orbitally. Samples were incubated at $37^{\circ} \mathrm{C}$ for $30 \mathrm{~min}$ before sera were collected and stored at $-70^{\circ} \mathrm{C}$. Lung tissues were rapidly dissected from mice and cryopreserved in liquid nitrogen for use in West- ern blotting and RT-PCR. Bronchoalveolar lavage fluid was collected by exposing the pars cervicalis trachea and incising a "V" in the lower trachea. Lower trachea was intubated with $24 \mathrm{G}$ needle. Saline $(0.9 \mathrm{ml})$ was gently injected and pumped back 3 times for irrigation to recover $>90 \%$ of BALF for leukocyte count/classification and cytokine detection. Spleens were collected for analysis of $\mathrm{T}$ cell ratios.

\section{Bronchoalveolar lavage fluid total cell number and leukocyte classification}

Bronchoalveolar lavage fluid $(6 \mu \mathrm{l})$ was applied to a counting plate (Rotork Instruments, Winston-Salem, NC, USA). Leukocytes were counted in the 4-angle square under $10 \times$ magnification on a light microscope. The leukocyte number was determined according to the formula: N/4 $\times 10^{6}$. The remaining BALF was centrifuged $\left(4^{\circ} \mathrm{C}, 1500\right.$ $\mathrm{rpm}, 10 \mathrm{~min}$ ), then stained with Wright Giemsa staining. Sediment smears were evaluated under $40 \times$ magnification on a light microscope. A minimum of 200 cells per sample were classified using a single-blind method to identify eosinophils, monocytes, and lymphocytes. Each test was repeated once.

\section{Expression of TGF- $\beta 1$, IL-10 and IL-17 in bronchoalveolar lavage fluid}

ELISA kits (R\&D Systems, USA) were used according to the manufacturer's instructions to detect the secreted levels of cytokines transforming growth factor $\beta 1$ (TGF- $\beta 1$ ), interleukin (IL) 10 and IL-17 in BALF. Each test was repeated once.

\section{Western blotting}

Lung tissue was treated with $150 \mu$ of the cell lysis solution $[0.1 \mathrm{mM} \mathrm{NaCl}, 0.01 \mathrm{mM}$ Tris- $\mathrm{HCl}(\mathrm{pH} 7.6)$ $0.001 \mathrm{mM}$ EDTA (pH 8.0), $1 \mu \mathrm{g} / \mathrm{ml}$ aprotinin, $100 \mu \mathrm{g} / \mathrm{ml}$ benzyl sulfonyl fluoride] before ultrasonication and centrifugation. Protein concentration was determined by Lowry method. Total protein $(30 \mu \mathrm{g})$ was separated by SDS-PAGE. Transfer printing, hybridization, ECL reaction inside a dark room, X-ray film developing, fixing and scanning the stripe of protein imprinting. Each test was repeated once.

\section{RT-PCR}

Lung tissue was processed using the Ultraspec TM-II RNA separation system (Biotecx Laboratories) to extract total RNA. RNA samples were reverse transcribed into cDNA for RT-PCR reaction using a kit (Takara, Japan) according to the manufacturer's instructions. Primers were designed used GenBank, and primers were synthesized by ShengGong Biological Engineering (Shanghai). Primer sequences and expected band sizes for Tlr4 and Gapdh (internal control) are shown in Table 1. Thermal cycling 
Table 1. PCR primer sequence and length of expected product

\begin{tabular}{lcc}
\hline Primer & Sequence & PCR product (bp) \\
\hline Tlr4 & AAACTTGCCTTCAAAACCTGGC & 356 \\
\cline { 2 - 3 } Gapdh & ACCTGAACTCATCAATGGTCA & 695 \\
\cline { 2 - 3 } & GGGAAGCTCATCGGCATGG & \\
\hline
\end{tabular}

conditions were as follows: $94^{\circ} \mathrm{C}$ for $45 \mathrm{~s}, 52^{\circ} \mathrm{C}$ for $60 \mathrm{~s}$, and $72^{\circ} \mathrm{C}$ for $90 \mathrm{~s}$ for 30 cycles; final extension at $72^{\circ} \mathrm{C}$ for 5 min. Products $(10 \mu \mathrm{l})$ were separated on $1.5 \%$ agarose gel and visualized with ethidium bromide staining. Image analysis software was used to determine the optical density ratio of the Tlr4 bands relative to Gapdh for relative expression of mRNA. Each test was repeated once.

\section{Flow cytometry}

Spleens were forced through 200-well metal mesh. Cells were collected and washed with cold RPMI-1640 (Gibco, USA) twice $(500 \times$ g centrifugation, $5 \mathrm{~min})$. Red blood cell lysate $(1 \mathrm{ml})$ was added. After incubation for 10 min at room temperature, samples were centrifuged again and supernatants were discarded. Cell concentration was adjusted to $8 \times 10^{7}$ with RPMI-1640 containing $10 \%$ fetal bovine serum.

Samples were split into 2 Eppendorf tubes. One tube was inoculated in a 6-well culture plate, to which phorbol myristate acetate $(50 \mathrm{ng} / \mathrm{ml})$, ionomycin $(500 \mathrm{ng} / \mathrm{ml})$, and brefeldin A ( $2 \mu \mathrm{g} / \mathrm{ml}$, Caltag, USA) were added. Plates were incubated at $37^{\circ} \mathrm{C}$ and $5 \% \mathrm{CO}_{2}$ for $4 \mathrm{~h}$. Cells were collected into an Eppendorf tube and washed with dyeing buffer solution. After centrifugation as before, supernatants were discarded. Anti-CD4-FITC (BD Pharmingen, USA) was added to each sample for $20 \mathrm{~min}$ at room temperature in the dark. Next, the dyeing buffer solution was added, and, after one washing, samples were centrifuged again and supernatants were discarded. $1 \mathrm{ml}$ of freshly prepared fixation/permeabilization solution (Caltag, USA) was added and samples were incubated for $30-60 \mathrm{~min}$ at $4{ }^{\circ} \mathrm{C}$ in the dark. Samples were then washed twice with $2 \mathrm{ml}$ of $1 \times$ permeabilization buffer (eBiosciences, USA), then $100 \mu \mathrm{l}$ of $1 \times$ permeabilization buffer (eBiosciences, USA) were added for $20 \mathrm{~min}$ at room temperature in the dark. Samples were centrifuged as before and supernatants were removed. Anti-IL-17-PE (BD, Becton Drive, NJ USA) was added and samples were incubated for $30 \mathrm{~min}$ at room temperature in the dark. After washing with $2 \mathrm{ml}$ of $1 \times$ permeabilization buffer, $500 \mu \mathrm{l}$ of PBS were added to resuspend cells. Samples were then used in flow cytometry.

In the second tube of tissue, $20 \mu 1$ of CD4-FITC and $20 \mu \mathrm{l}$ of CD25-PE (BD Pharmingen, USA) were added for incubation for $30 \mathrm{~min}$ at $4{ }^{\circ} \mathrm{C}$ away from light. After washing with cold PBS, $1 \mathrm{ml}$ of freshly prepared fixation/per- meabilization solution was added for incubation for 30-60 min at $4^{\circ} \mathrm{C}$ away from light. Samples were washed twice with $2 \mathrm{ml}$ of $1 \times$ permeabilization buffer, then resuspended in $100 \mu$ l of $1 \times$ permeabilization buffer and incubated for $20 \mathrm{~min}$ at room temperature away from light. Samples were centrifuged as before and supernatants were discarded. Anti-Foxp3-APC antibody (Teterow, Germany) was added and samples were incubated at $4^{\circ} \mathrm{C}$ for $30 \mathrm{~min}$ in the dark. Samples were washed with $2 \mathrm{ml}$ of $1 \times$ permeabilization buffer, then resuspended in $500 \mu$ of PBS for flow cytometry. Each test was repeated once.

\section{Statistical methods}

Data were analyzed with SPSS17.0. Measurements are presented as mean \pm standard deviation $(\bar{X} \pm \mathrm{s})$. Monofactorial analysis of variance (ANOVA) and SNK method were used to compare groups. $P<0.05$ was accepted as statistically significant.

\section{Results}

\section{Pathological changes in lung tissue}

In the $\mathrm{CT}$ group, the pulmonary bronchioles were complete, and there was no abnormality in the pulmonary wall or muscle. In AST group, there were some damages in bronchial wall, absent in epithelial, and results showed thickened capillaries in the bronchial wall with increased inflammatory exudates and infiltration. After treatment by Dynasore, the mice had significant changes, and the severity of inflammatory cell infiltration in bronchi, bronchioles and accompanying the vessels decreased, and the edema improved, seen in Fig. 1.

\section{Dynasore treatment alters blood cell distributions in bronchoalveolar lavage fluid}

To determine how white blood cell distributions are affected in mice with asthma that have been treated with TRIF inhibitor, cell counts were determined in CT, AST, and DYN groups. Mice with induced asthma displayed different distributions of blood cells in BALF. Indeed, the total number of white blood cells, eosinophils, monocytes, and lymphocytes were significantly higher in BALF of the AST group than of the control group $(p<0.05$; Table 2). However, when mice with induced asthma were treated with 

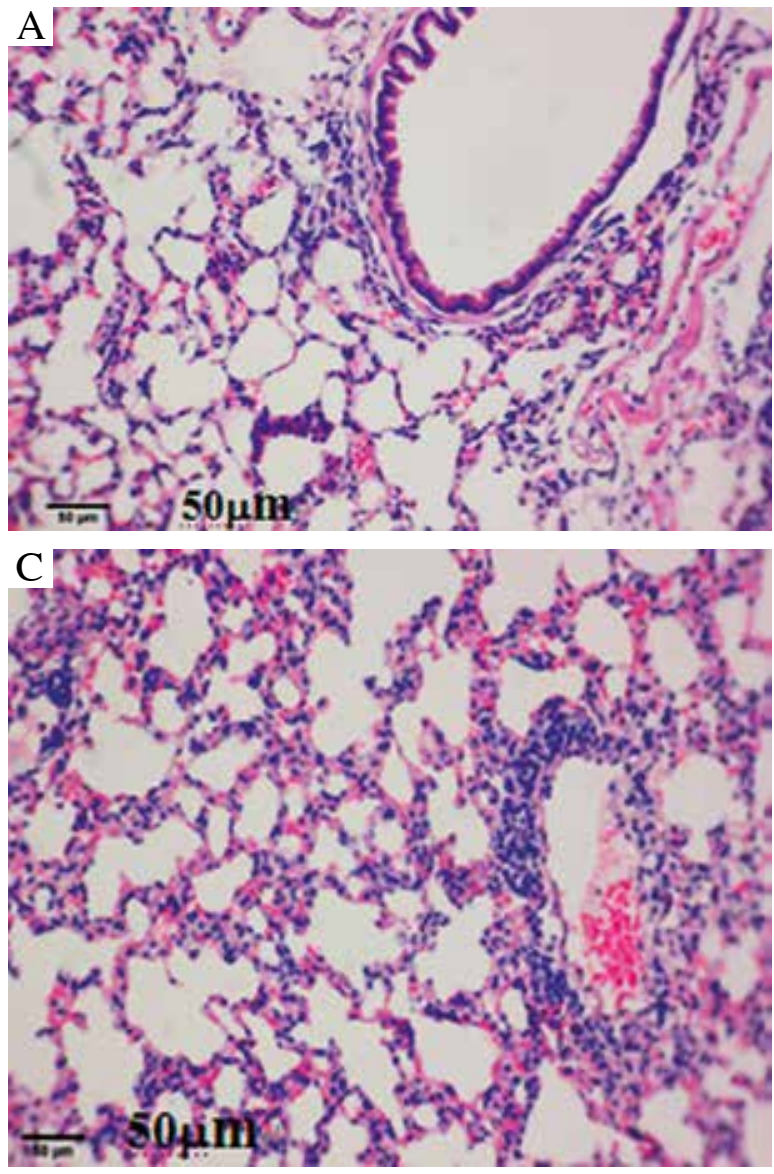

Dynasore to inhibit TLR4/TRIF signaling (DYN group), the total number of white blood cells, eosinophils, monocytes, and lymphocytes in BALF were significantly decreased compared to untreated mice with asthma $(p<0.05)$.

\section{Varying levels of secreted cytokines IL-10 and IL-17 in bronchoalveolar lavage fluid}

Cytokines IL-10 and IL-17 are indicative of anti-inflammatory and pro-inflammatory processes, respectively. Therefore, secretion levels of these cytokines was assessed in BALF of our mouse models. Mice with induced asthma exhibited lower levels of secreted IL-10 but higher levels

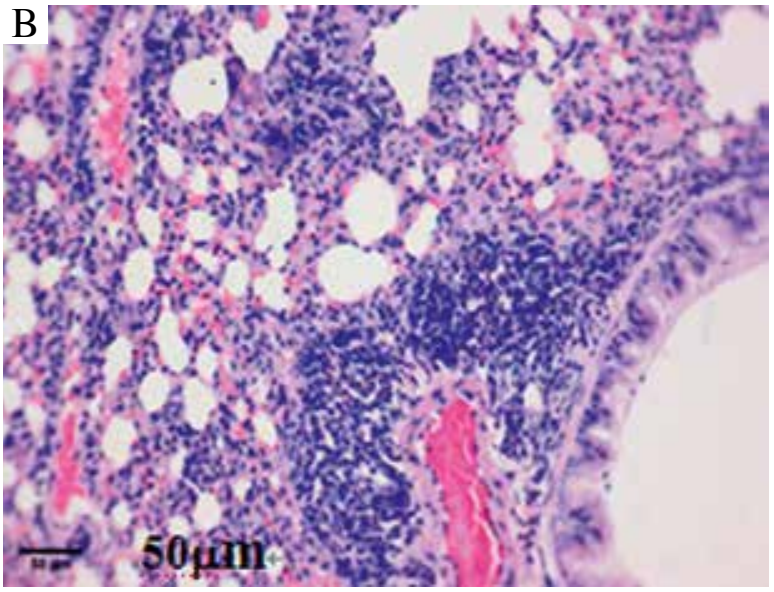

Fig. 1. The pathological changes in lung tissue. A) control group (CT group); B) asthma group (AST group); C) Dynasore treatment group (DYN group)

of secreted IL-17 in BALF compared with healthy control mice ( $p<0.05$; Table 3). However, inhibition of TLR4/ TRIF signaling with Dynasore treatment in mice with induced asthma displayed significantly increased levels of IL-10 and decreased levels of IL-17 in BALF $(p<0.05)$.

\section{Altered expression of Toll-like receptor 4 protein and mRNA in lung tissue}

To determine whether inhibition of TRIF altered TLR4 expression, a relative expression of TLR4 protein and mRNA was assessed in lung tissue. Mice with induced asthma had significantly higher levels of both protein and

Table 2. Total white cell and differential cell counts in BALF of mice $\left(\times 10^{6} / 1\right)$

\begin{tabular}{lccccc}
\hline Group & $\boldsymbol{n}$ & Total white cells & Eosinophil & Monocyte & Lymphocyte \\
\hline CT & 10 & $9.91 \pm 1.39$ & $0.03 \pm 0.01$ & $7.97 \pm 1.21$ & $1.91 \pm 0.58$ \\
\hline AST & 10 & $94.88 \pm 10.54^{*}$ & $15.07 \pm 1.75^{*}$ & $52.51 \pm 8.08^{*}$ & $27.31 \pm 5.59^{*}$ \\
\hline DYN & 10 & $38.95 \pm 8.54^{* \#}$ & $3.52 \pm 0.95^{* \#}$ & $28.39 \pm 7.93^{* \#}$ & 114.955 \\
\hline$F$ & & 300.958 & 467.778 & 0.001 & 150.894 \\
\hline$P$
\end{tabular}

CT - control, AST - asthma, DYN - Dynasore

"vs. NS group: $P<0.05$; $" v s$. AST group: $P<0.05$ 
mRNA expression than control mice ( $p<0.05$; Figs. 2, 3 ). Mice with induced asthma treated with Dynasore, however, exhibited decreases in the levels of both TRL4 protein and mRNA in lung tissue compared to untreated mice with asthma $(p<0.05)$.

\section{Treg/Th17 cell ratios change following TLR4/ TRIF inhibition}

To determine whether treatment of mice with induced asthma alters inflammatory responses by changing the Treg/Th17 ratio, we identified CD4+CD25+Foxp3+ (Treg) and CD4+IL-17+ (Th17) cells via flow cytometry of lymphocytes from spleen tissue. The proportion of Treg cells (from all CD4+ cells) was significantly lower while the proportion of Th17 cells was significantly higher in mice with induced asthma compared with control mice; thus, the Treg/Th17 ratio was significantly lower in AST mice $(P<0.05$; Table 4). However, Dynasore treatment of mice with induced asthma mice resulted in a significantly lower proportion of Treg cells and a significantly higher proportion of Th17 cells than in AST mice, resulting in a higher ratio of Treg/Th17 cells $(p<0.05)$.

\section{Discussion}

Airway pathologies characterizing bronchial asthma, including inflammation, hyper-responsiveness, and remodeling, result from activation of inflammatory cells, particularly eosinophils and lymphocytes, and release of inflammatory mediators [12]. This work developed the asthmatic model by OVA, results showed that the pulmonary bronchioles wall and epithelial cells were damaged, congestion and edema were observed in bronchioles and surrounding interstitial, inflammatory exudates filled cavity, a lot of inflammatory cell infiltration present from bronchiole walls. The induced model of asthma resulted in higher total numbers of white blood cells, particularly eosinophils, monocytes, and lymphocytes, in BLAF, suggesting that, as
Table 3. Concentration of IL-10 and IL-17 protein secreted in BALF (pg/ml)

\begin{tabular}{|c|c|c|c|}
\hline Group & $\mathbf{n}$ & IL-10 & IL-17 \\
\hline CT & 10 & $103.16 \pm 22.22$ & $5.75 \pm 1.35$ \\
\hline AST & 10 & $30.12 \pm 10.70 *$ & $12.01 \pm 3.63^{*}$ \\
\hline DYN & 10 & $68.37 \pm 20.76^{* \#}$ & $8.85 \pm 3.42 * \#$ \\
\hline$F$ & & 38.521 & 10.102 \\
\hline$P$ & & 0.001 & 0.001 \\
\hline
\end{tabular}

would be expected, mice in the asthma group had inflammatory cells infiltrating the airway.

Toll-like receptors are typically considered central to innate immunity [13]. The TLR family comprises at least 12 members. Toll-like receptor 4 is expressed in most cell lines, but is typically identified on the surface of cells acting in host defense, such as monocytes, granulocytes, dendritic cells, macrophages, lymphocytes, endothelial and epithelial cells; it is highly expressed on bone marrow mononuclear cells $[14,15]$. This receptor is critical to signal transduction in both MyD88-dependent and MyD88-independent pathways $[5,6]$. An adapter protein, TRIF is the main junction of MyD88-independent signal transduction. Toll-like receptor 4/TRIF signaling in chronic airway inflammation of asthma helps activate and differentiate $\mathrm{T}$ cells, as well as stimulating their cytokine production [7]. Here, both protein and mRNA expression levels of TLR4 were higher in lung tissue of mice with induced asthma, suggesting a link between TLR4/TRIF signaling and asthma pathogenesis.

Th17 cells secrete IL-17 to promote inflammatory response; these cells are associated with asthma pathology [16]. In contrast, Treg cells produce anti-inflammatory cytokines like IL-10 and TGF- $\beta$ [16]. In asthma patients, declines in Treg numbers signal worsening disease [17]; when animal models of asthma are infused with Treg cells
NS

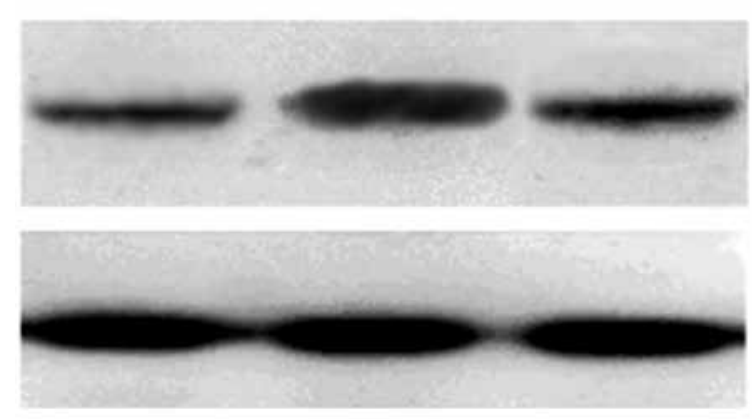

M NS AST DYN

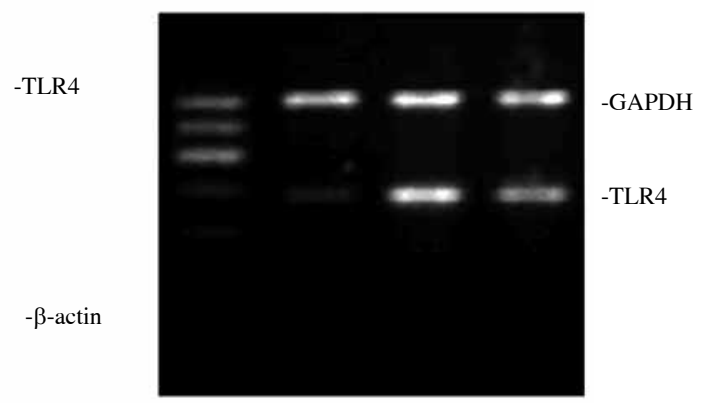

Fig. 2. Protein (A) and mRNA (B) expression of TLR4 in lung tissue 
Table 4. Percentage of Treg and Th17 cells isolated from spleen tissue by flow cytometry

\begin{tabular}{lcccc}
\hline Group & $\mathbf{n}$ & CD4+CD25+Foxp3+ cell & CD4+IL-17+ cell & Treg/Th17 ratio \\
\hline CT & 10 & $14.39 \pm 1.47$ & $0.64 \pm 0.06$ & $22.04 \pm 1.87$ \\
AST & 10 & $5.50 \pm 1.45^{*}$ & $1.45 \pm 0.36^{*}$ & $4.16 \pm 2.09^{*}$ \\
DYN & 10 & $9.91 \pm 1.91^{* \#}$ & $1.02 \pm 0.30^{* \#}$ & $10.94 \pm 5.18^{* *}$ \\
$F$ & 74.715 & 21.416 & 73.557 \\
$P$ & & 0.001 & 0.001 & 0.001 \\
\hline
\end{tabular}

CT - control, AST - asthma, DYN- Dynasore

"vs. NS group: $P<0.05$; $*$ vs. AST group: $P<0.05$.

A

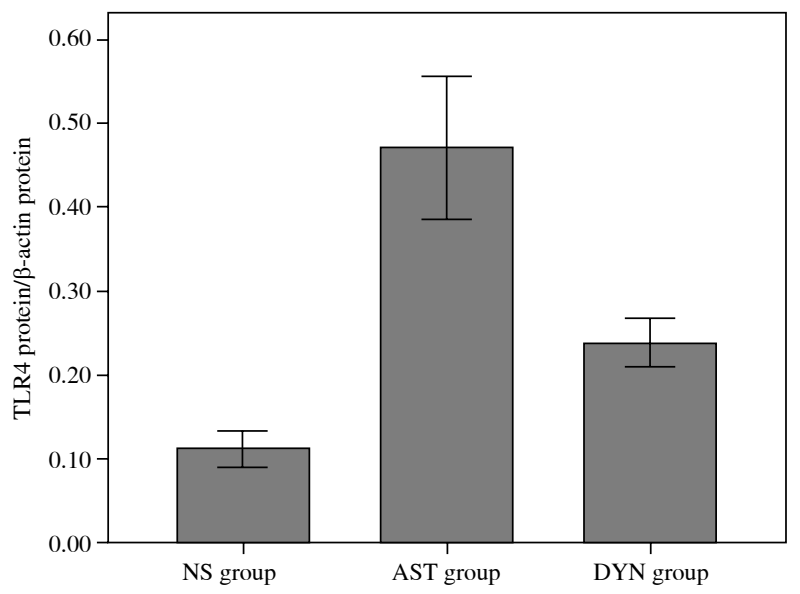

B

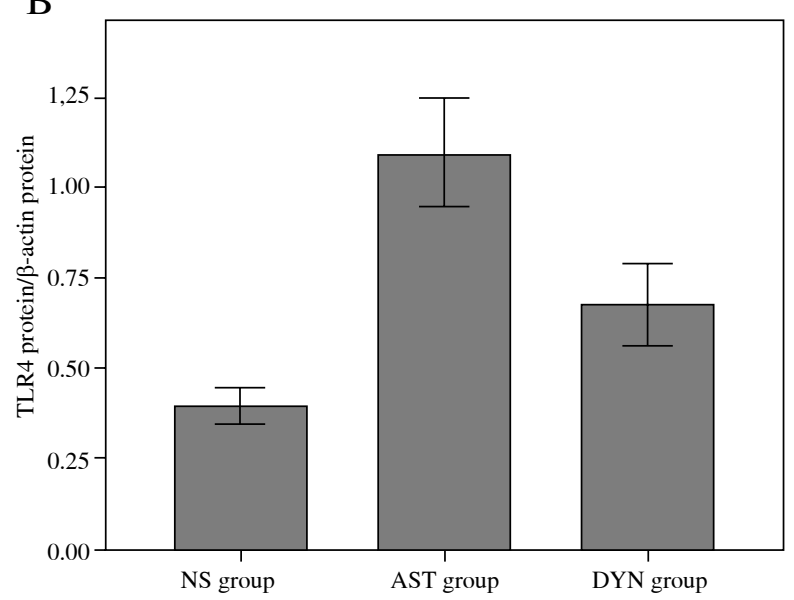

Fig. 3. Relative expression of TLR4 protein (A) and mRNA (B) in lung tissue

cultured in vitro, airway hyper-responsiveness, acidophilic granulocyte infiltration, and Th2 cytokine production decrease [18]. Imbalances in the ratio of Treg/Th17 cells have been identified in asthma patients [19]. Here, the proportions of Treg and Th17 subsets of lymphocytes were altered in mice with induced asthma, leading to an altered (lower) Treg/Th17 ratio and implying an association of changes in these lymphocytes with inflammation.

Dynasore, a specific compound of dyneins (dynamin), inhibits $\mathrm{G}$ protein activity of Dynamin, affecting physiological functions such as cellular endocytosis. Although it has no effect on protein transport from endoplasmic reticulum to the Golgi apparatus in mammalian cells in vitro, it inhibits liquid-phase pinocytosis and endocytosis. Thus, Dynasore inhibits dynamin; because TLR4/TRIF signaling promotes endocytosis mainly by Dynamin, it is hypothesized that Dynasore blocks TLR4/TRIF signaling $[10,11]$.

Here, Dynasore was used to block TLR4/TRIF signaling in mice with induced asthma to determine how inhibition of the signal cascade could affect inflammatory responses. Compared to mice with induced asthma that were untreated, Dynasore intervention resulted in lower white blood cell, eosinophil, monocyte, and lymphocyte counts in BALF, as well as a decreased TLR4 protein and mRNA expression in the lung tissue. Further, the subsets of Treg and Th17 lymphocytes were altered in DYN mice compared to AST mice, with more Treg and fewer Th17 cells, indicating higher anti-inflammatory and lower pro-inflammatory processes. This was supported by an increased secretion of IL-10 and decreased secretion of IL-17 in BALF. These results indicate that Dynasore suppresses TLR4/TRIF downstream signaling and influences the release of inflammatory mediators and inflammatory cells infiltration. Thus, we could expect that airway inflammation and remodeling could be ameliorated by this intervention.

This study was supported by Grants of the Zhejiang Provincial Natural Science Foundation of China (No. LY12H10003) and the Zhejiang Provincial Medical and Health Foundation of China (No. 2013KYB303). 


\section{References}

1. Lommatzsch M. (2012): Airway hyperresponsiveness: New insights into the pathogenesis. Semin Respir Crit Care Med 33: 579-587.

2. Awasthi A, Murugaiyan G, Kuchroo VK (2008): Interplay between effector Th17 and regulatory T cells. J Clin Immunol 28: 660-670.

3. Palomares O, Yaman G, Azkur AK, et al. (2010): Role of Treg in immune regulation of allergic diseases. Eur J Immunol 40: 1232-1240.

4. Trompette A, Divanovic S, Visintin A, et al. (2009): Allergenicity resulting from functional mimicry of a Toll-like receptor complex protein. Nature 457: 585-588.

5. Georgel P, Jiang Z, Kunz S, et al. (2007): Vesicular stomatitis virus glycoprotein $\mathrm{G}$ activates a specific antiviral Toll-like receptor 4-dependent pathway. Virology 362: 304-313.

6. Lee JK, Kim SY, Kim YS, et al. (2009). Suppression of the TRIF-dependent signaling pathway of Toll-like receptors by luteolin. Biochem Pharmacol 77: 1391-1400.

7. Ve T, Gay NJ, Mansell A, et al. (2012): Adaptors in toll-like receptor signaling and their potential as therapeutic targets. Curr Drug Targets 13: 1360-1374.

8. Biswas SK, Bist P, Dhillon MK, et al. (2007): Role for MyD88-independent, TRIF pathway in lipid A/TLR4-induced endotoxin tolerance. J Immunol 179: 4083-4092.

9. Brown J, Wang H, Hajishengallis GN (2011): TLR-signaling networks: an integration of adaptor molecules, kinases, and cross-talk. J Dent Res 90: 417-427.

10. Douthitt HL, Luo F, McCann SD, Meriney SD (2011): Dynasore, an inhibitor of dynamin, increases the probability of transmitter release. Neuroscience 172: 187-195.

11. Kadoki M, Choi BI, Iwakura Y (2010): The mechanism of LPS-induced HIV type I activation in transgenic mouse macrophages. Int Immunol 22: 469-478.

12. Hirota JA, Hackett TL, Inman MD, Knight DA (2011): Modeling asthma in mice: what have we learned about the airway epithelium. Am J Respir Cell Mol Biol 44: 431-438.

13. Medzhitov R, Preston-Hurlburt P, Janeway CA Jr (1997): A human homologue of the drosophila Toll protein signals activation of adaptive immunity. Nature 388: 394-397.

14. Janeway CA Jr, Medzhitov R (2002): Innate immune recognition. Annu Rev Immunol 20: 197-216.

15. Gioannini TL, Weiss JP (2007): Regulation of interactions of Gram-negative bacterial endotoxins with mammalian cells. Immunol Res 39: 249-260.

16. Wong CK, Ho CY, Ko FW, et al. (2001): Proinflammatory cytokines (IL-17, IL-6, IL-18 and IL-12) and Th cytokines (IFN- $\gamma$, IL-4, IL-10 and IL-13) in patients with allergic asthma. Clin Exp Immunol 125: 177-183.

17. Sakaguchi S, Sakaguchi N, Asano M, et al. (1995): Immunologic self-tolerance maintained by activated T cells expressing IL-2 receptor alpha-chains (CD25). Breakdown of a single mechanism of self-tolerance causes various autoimmune diseases. J Immunol 155: 1151-1164.

18. Kearley J, Barker JE, Robinson DS, Lloyd CM (2005). Resolution of airway inflammation and hyperreactivity after in vivo transfer of CD4+CD25+ regulatory T cells is interleukin 10 dependent. J Exp Med 202: 1539-1547.

19. Shi YH, Shi GC, Wan HY (2010): The prevalence of blood Th17 and CD4+CD25+Treg cells in patients with bronchial asthma. Chinese Journal of Immunology 26: 740-743 (in Chinese). 